Published in final edited form as:

Am J Med. 2011 December ; 124(12): 1175.e9-1175.17. doi:10.1016/j.amjmed.2011.05.029.

\title{
Processes of Care and Outcomes for Community-Acquired Pneumonia
}

\author{
Jonathan S. Lee, BS ${ }^{a}$, Brian A. Primack, MD, EdM, MS ${ }^{a, b, c}$, Maria K. Mor, PhDe,f, Roslyn A. \\ Stone, PhD ${ }^{b, e}$, , D. Scott Obrosky, MSc $^{e}$, Donald M. Yealy, MD $^{d}$, and Michael J. Fine, MD, \\ $M c^{a, b, e}$ \\ aDivision of General Internal Medicine, Department of Medicine, University of Pittsburgh School of \\ Medicine, Pittsburgh, Penn \\ ${ }^{b}$ Center for Research on Health Care, University of Pittsburgh School of Medicine, Pittsburgh, \\ Penn \\ 'Division of Adolescent Medicine, Department of Pediatrics, University of Pittsburgh School of \\ Medicine, Pittsburgh, Penn \\ dDepartment of Emergency Medicine, University of Pittsburgh School of Medicine, Pittsburgh, \\ Penn \\ eVA Center for Health Equity Research and Promotion, VA Pittsburgh Healthcare System, \\ Pittsburgh, Penn \\ fDepartment of Biostatistics, University of Pittsburgh Graduate School of Public Health, \\ Pittsburgh, Penn
}

\section{Abstract}

BACKGROUND-Although processes of care are common proxies for health care quality, their associations with medical outcomes remain uncertain.

METHODS-For 2076 patients hospitalized with pneumonia from 32 emergency departments, we used multilevel logistic regression modeling to assess independent associations between patient outcomes and the performance of 4 individual processes of care (assessment of oxygenation, blood cultures, and rapid initiation $[<4$ hours] and appropriate selection of antibiotic therapy) and the cumulative number of processes of care performed.

RESULTS-Overall, 141 patients (6.8\%) died. Mortality was $0.3 \%$ to $1.7 \%$ lower for patients who had each of the individual processes of care performed ( $P \geq .13$ for each comparison); mortality was $7.5 \%$ for patients who had 0 to 2 processes of care, $7.2 \%$ for those with 3 processes of care, and $5.8 \%$ for those with all 4 processes of care performed $(P=.39)$. Mortality was not significantly associated with either individual or cumulative process measures in multivariable models.

CONCLUSION-Neither the individual processes of care nor the cumulative number performed is associated with short-term mortality for pneumonia.

Requests for reprints should be addressed to Michael J. Fine, MD, MSc, Veterans Administration Pittsburgh Healthcare System (151

C-H), Center for Health Equity Research and Promotion, 7180 Highland Drive, Room 4059E, Pittsburgh, PA 15206.

Michael.Fine@va.gov.

Authorship: All authors had access to the data and played a role in writing this manuscript.

Conflict of Interest: None. 


\section{Keywords}

Pneumonia; Processes of care; Quality of care

Over the past decade, evidence-based processes of care have been the primary targets of national efforts to improve the quality of care for patients with pneumonia. ${ }^{1-6}$ The Joint Commission on Accreditation of Healthcare Organizations uses these measures in its national hospital accreditation program, and the Centers for Medicare \& Medicaid Services (CMS) link them to public reporting and pay-for-performance initiatives.$^{7-10}$ Although secular improvements in mortality, hospital length of stay, and readmission have been documented during a similar time frame as improvements in hospital performance of processes of care, ${ }^{11-13}$ direct associations between improved performance of pneumoniaspecific processes of care and patient outcomes remain unclear.

Although some observational studies have demonstrated significant associations between performance of blood cultures, rapid initiation of antibiotic therapy, appropriate selection of antibiotic therapy, and improved outcomes for patients hospitalized with pneumonia, ${ }^{14-19}$ not all studies have demonstrated these results. ${ }^{20-22}$ The majority of these studies were retrospective, ${ }^{15-20}$ small, ${ }^{14,19,21}$ or conducted at a single site, ${ }^{14,21}$ and had imperfect adjustment for severity of illness at presentation and limited assessment of patient-centered medical outcomes. To our knowledge, no studies have reported dose-response associations between the number of recommended processes of care performed and patient outcomes. We studied the associations between the performance of evidence-based processes of care and short-term mortality and other medical outcomes in patients hospitalized with pneumonia in the Emergency Department Community-Acquired Pneumonia (EDCAP) Trial. $^{23}$

\section{MATERIALS AND METHODS \\ Study Design and Patient Eligibility}

We studied hospitalized patients enrolled in a multicenter, cluster-randomized study performed at hospital emergency departments in Connecticut $(\mathrm{n}=16)$ and Pennsylvania $(\mathrm{n}=$ 16) between January and December $2001 .{ }^{23}$ Study sites were randomized to low-intensity (n $=8)$, moderate-intensity $(\mathrm{n}=12)$, or high-intensity $(\mathrm{n}=12)$ guideline implementation strategies to promote performance of evidence-based processes of care for pneumonia and to use the Pneumonia Severity Index (PSI) to guide the initial site of treatment decision. The PSI is a validated prediction rule for pneumonia prognosis that stratifies patients into 5 classes of risk for mortality. ${ }^{24}$ Institutional review boards responsible for all sites approved the trial. EDCAP study methods and findings on the interventions' effectiveness and safety have been reported. ${ }^{23,25}$

We enrolled adult patients (aged $\geq 18$ years) with clinical and radiographic evidence of pneumonia. We excluded patients with hospital-acquired pneumonia, immunosuppression, specified conditions (eg, cystic fibrosis, pregnancy), or psychosocial or substance abuse problems incompatible with outpatient treatment or follow-up.

We defined inpatient treatment as hospital admission, transfer from an emergency department to an inpatient hospital observation unit, or admission to an emergency department observation unit with discharge to any setting more than 24 hours after presentation. The current study focuses exclusively on inpatients, because most data linking processes of care and outcomes for pneumonia come from such populations, ${ }^{14-19}$ and the 
increased severity of illness among inpatients enhanced our opportunity to detect processoutcome associations.

\section{Practice Guideline Implementation}

The practice guideline used in the EDCAP Trial consisted of 4 recommendations for initial processes of care for patients hospitalized from the emergency department: assess arterial oxygenation on presentation; obtain 2 blood cultures before antibiotic administration; initiate antibiotic therapy within 4 hours of presentation; and appropriately select empiric antibiotic therapy. All 4 processes of care were recommended by medical society guidelines preceding the trial. ${ }^{5}$

\section{Baseline Data Collection}

We collected data on patient demographics, comorbid conditions, physical examination findings, laboratory and radiographic findings, and medical treatments. We also collected information on emergency department medical providers and participating emergency departments.

\section{Performance of Processes of Care}

We documented the performance of the guideline-recommended processes of care by reviewing medical records. We defined the assessment of arterial oxygenation as the performance of pulse oximetry or an arterial blood gas in the emergency department. We defined administration of appropriate antibiotic therapy as the prescription of guidelinerecommended drug(s) for patients admitted to a hospital ward, an intensive care unit, or coronary care unit from the emergency department. We determined whether blood cultures were performed before initiation of antibiotic therapy. The time interval between presentation and initiation of antibiotic therapy denoted whether the first dose of antibiotic therapy was received within 4 hours. We created a categoric summary measure $(0-2,3$, and 4) of the total number of individual processes of care performed.

\section{Patient Outcomes}

Our primary study outcome was mortality 30 days after presentation. During the index hospitalization, we assessed length of stay in days (median and interquartile range) and intensive care unit or coronary care unit admissions for cardiac ischemia or arrhythmia, respiratory failure, or hemodynamic compromise. We also assessed readmission within 30 days after initial presentation.

\section{Statistical Analyses}

We used chi-square and Kruskal-Wallis tests to compare baseline patient characteristics. We used the Rao and Scott second-order corrected Pearson chi-square test statistic to compare provider and site characteristics across the number of processes of care performed, accounting for the clustering of patients at the provider and site levels..$^{26,27}$

To assess associations between process measures and patient outcomes, we used multilevel logistic regression modeling to account for the clustering of patients within providers and sites. For length of stay analyses, these multilevel models were calculated using discrete proportional hazards models, with patients who died during the index hospitalization censored on death. We adjusted for baseline severity of illness using PSI risk class and patient, provider, and site characteristics not included in the PSI (listed in Table 1).

To allow comparisons to studies conducted in Medicare populations, ${ }^{15-18}$ we used similar methods to analyze the multivariable associations between process measures and patient 
outcomes for patients aged 65 years or more. Because admission to an intensive care unit or coronary care unit from the emergency department affects the definition of appropriate antibiotic therapy, we also assessed the effects of such admissions and their timing (ie, on or after the day of presentation) on associations between process measures and patient outcomes.

We conducted all statistical analyses using Stata 11 (StataCorp LP, College Station, Tex) and MLwiN 2.02 (University of Bristol, Bristol, UK). Two-sided $P$ values $<.05$ were considered statistically significant.

\section{RESULTS}

We enrolled 3615 (80.2\%) of 4506 eligible patients and excluded 414 patients from process of care analyses. This study focuses on the 2076 hospitalized patients in the EDCAP Trial.

\section{Patient, Provider, and Site Characteristics}

The median age of patients was 74 years, $51.2 \%$ were women, $90.3 \%$ were non-Hispanic white, and 51.5\% were in the 3 lowest PSI risk classes (Table 1). Of the 378 responsible medical providers, $81.0 \%$ were male and $67.0 \%$ worked 12 or more shifts per month. Most $(90.6 \%)$ of the 32 emergency departments were located in urban areas, and $50.0 \%$ were affiliated with teaching hospitals.

\section{Performance of Processes of Care}

Overall, 2027 patients (97.6\%) had an assessment of arterial oxygenation, 1314 patients $(63.3 \%)$ received 2 blood cultures before antibiotic therapy, 1632 patients $(78.6 \%)$ received the first antibiotic dose within 4 hours, and 1308 patients (63.0\%) received appropriate antibiotic therapy (Table 2). Cumulatively, 534 patients (25.7\%) received 0 to 2 processes of care, 837 patients $(40.3 \%)$ received 3 processes of care, and 705 patients (34.0\%) received all 4 recommended processes of care. Patients admitted to an intensive care unit or coronary care unit on the day of admission were less likely to receive appropriate antibiotic therapy compared with patients never admitted to such a unit or admitted $\geq 1$ day after hospitalization $(40.4 \%, 64.8 \%$, and $60.9 \%$, respectively; $P<.001)$.

\section{Patient Outcomes}

A total of 141 patients (6.8\%) died within 30 days, 275 patients (13.3\%) were admitted to an intensive care unit or coronary care unit during the index hospitalization (141 on day of admission), and 175 patients (8.8\%) were rehospitalized within 30 days of presentation. Overall, the median length of stay was 5 days (interquartile range 3-7).

Mortality was higher in the 1476 patients aged 65 years or more compared with younger patients $(8.6 \%$ vs $2.4 \%, P<.001)$ and in the 275 patients who were ever (versus never) treated in an intensive care unit or coronary care unit $(18.2 \%$ vs $5.1 \%, P<.001)$. Median length of stay was longer for patients aged 65 years or more compared with younger patients (median 5 days, interquartile range $3-8$ vs median 4 days, interquartile range $3-6 ; P<.001$ ). None of the study outcomes differed significantly by intervention arm.

\section{Associations of Processes of Care and Mortality}

As shown in Table 3, mortality was $0.3 \%$ to $1.7 \%$ lower for patients who had each of the individual processes of care performed compared with those who did not ( $P \geq 13$ for each). Mortality was $7.5 \%$ and $7.2 \%$ for patients who had 0 to 2 and 3 processes of care performed, respectively, compared with $5.8 \%$ for patients who had all 4 performed $(P=.39)$. The 
adjusted odds ratios (ORs) for mortality were nonsignificantly reduced for each process of care (ranging from 0.7 to $0.9 ; P \geq 13$ for each).

\section{Associations of Processes of Care and Secondary Outcomes}

The unadjusted point estimates for secondary patient outcomes are provided in Table 4 . The adjusted ORs (Table 5) of the independent associations between individual processes of care and cumulative number performed and the secondary patient outcomes are consistent with the point estimates. Performance of blood cultures before antibiotic therapy was associated with an increased adjusted odds of intensive care unit or coronary care unit admission (OR, 1.4; 95\% confidence interval [CI], 1.0-1.9); antibiotic administration within 4 hours was associated with a decreased adjusted odds for length of stay (OR, 1.2; 95\% CI, 1.1-1.4); and appropriate antibiotic therapy was associated with a decreased adjusted odds of intensive care unit or coronary care unit admission (OR, 0.5 ; 95\% CI, 0.4-0.7). Patients who had 4 processes of care performed had a decreased adjusted odds for length of stay relative to those with 0 to 2 processes of care performed (OR, 1.2; 95\% CI, 1.1-1.4).

\section{Subgroup Analyses}

None of the individual processes of care or the cumulative number performed was significantly associated with 30-day mortality in patients aged 65 years or more. The magnitude of the associations between antibiotic administration within 4 hours and the number of processes of care performed and decreased length of stay were similar across both age strata.

For patients treated on a medical ward for 1 or more days after hospitalization, there were no independent associations between appropriate antibiotic therapy and length of stay (OR, 1.1; 95\% CI, 0.95-1.2) or subsequent intensive care unit or coronary care unit admission (OR, $0.8 ; 95 \% \mathrm{CI}, 0.5-1.1)$. For patients never treated in an intensive care unit or coronary care unit, antibiotic administration within 4 hours and the number of processes of care performed both remained independently associated with a decreased length of hospital stay.

\section{DISCUSSION}

In this multicenter study of 2076 patients hospitalized with pneumonia, we found no associations between 30 -day mortality and 4 commonly recommended evidence-based processes of care. Despite recent consensus medical specialty society guidelines recommending that local implementation of guidelines address a comprehensive set of processes of care with the goal of improving patient outcomes, ${ }^{3}$ we also were unable to identify any associations between the cumulative number of processes of care performed and mortality. Neither did we find associations between the individual or cumulative process measures and the 30-day hospital readmission rate, an outcome systematically tracked by the CMS as a quality metric for US hospitals. ${ }^{28}$

Although our observed association between rapid initiation of antibiotic therapy and decreased length of stay is consistent with the results of previous studies, ${ }^{16,19}$ the mechanism underlying this association remains unclear. At the patient level, it is possible that rapid antibiotic therapy leads to more rapid physiologic return to clinical stability and readiness for hospital discharge. At the provider level, clinicians who are compliant with this recommended process of care may more efficiently manage patients hospitalized for pneumonia. At the system level, hospitals that endorse the performance of such guidelinerecommended processes of care potentially have organizational cultures or structures that maximize efficiency of care. 
Two observed independent associations between individual processes of care (ie, performance of blood cultures and appropriate selection of antibiotic therapy) and intensive care unit or coronary care unit admission in our study are most likely explained by unmeasured confounding or selection bias. Clinicians' decisions to obtain blood cultures more frequently among patients admitted to an intensive care unit or coronary care unit likely reflect their assessments of increased severity of illness or their clinical suspicion of sepsis. The association of appropriate antibiotic selection with a decreased rate of intensive care unit or coronary care unit admission likely reflects the lower frequency of compliance with guideline-recommended antibiotic therapy among patients who were admitted to such a unit on the day of hospitalization, because of guideline recommendations that require broader-spectrum antibiotic regimens for this subgroup. That this association did not exist in the 1925 patients treated on a medical ward for the first day of hospitalization (of whom 133 subsequently required intensive care unit or coronary care unit admission) supports this explanation and repudiates the argument that appropriate antibiotic selection leads to decreased rates of subsequent intensive care unit or coronary care unit admission.

Our inability to detect associations between performance of processes of care and mortality is consistent with some previous studies, ${ }^{20,22}$ but inconsistent with others. ${ }^{14-18}$ We suggest 3 possible reasons. First, in contrast with most previous positive studies that focused on patients aged 65 years or more, ${ }^{15-18}$ our study included all adults. It is possible that the salutary effects of these processes of care are larger in magnitude and more discernable in elderly patients. However, our subgroup analysis of patients aged 65 years or more did not reveal any significant associations between processes of care and mortality. Second, it is possible that we adjusted more comprehensively for potential confounders of processoutcome associations by controlling for baseline severity of illness with the PSI and a wider array of patient, provider, and site-level covariates. Third, our study was not designed to assess process-outcome associations, and it may have lacked sufficient power to detect the observed $0.3 \%$ to $1.7 \%$ differences in mortality for individual process measures in 2076 patients. Most of the prior positive studies demonstrating significant process-outcome associations had sample sizes exceeding 10,000 patients. ${ }^{15-18}$ In fact, for a process measure with a baseline performance rate of $80 \%$ in a cohort with a baseline mortality of $7 \%$, a cohort of 38,000 would be required to detect a $1 \%$ mortality difference with $80 \%$ power.

The lack of observed associations between recommended process measures and mortality or hospital readmission, coupled with the observation that many of these recommended processes of care already have reached or are swiftly approaching ceiling levels of performance, raises questions about their continued use as proxy measures of health care quality for pneumonia. For example, assessment of arterial oxygenation at presentation was "retired" as a quality measure in 2009 by the CMS because it was performed in more than $99 \%$ of patients hospitalized for pneumonia. ${ }^{29}$ Data from 2010 indicate that the remaining 7 pneumonia performance measures are all performed in more than $90 \%$ of hospitalized patients. ${ }^{30}$ The use of aspirin and beta-blockers for patients after acute myocardial infarction or angiotensin-converting enzyme inhibitors for patients with chronic heart failure is supported by evidence from large randomized, controlled trials, and meta-analyses showing survival benefits for these processes of care. ${ }^{31-33}$ Unfortunately, similar high-quality evidence does not exist for pneumonia.

\section{STUDY LIMITATIONS}

Our study has several limitations. First, the study population was limited to inpatients, whereas the majority of patients with pneumonia are treated as outpatients. Because outcomes are so favorable in outpatients, it would not have been possible to assess associations between process measures and mortality in that subgroup. Second, our study is 
a secondary analysis of data from a trial to assess the effectiveness and safety of guideline implementation. It is possible that the exclusion criteria in the parent study limit the generalizability of our findings. Third, performance of the process measures in our study was at the discretion of the managing physicians, making it more difficult to assess nonconfounded process-outcome associations. However, our adjustment for pneumonia severity of illness and multiple additional potential confounding variables likely represents the best approach to understanding associations between processes of care and patient outcomes in the absence of randomized controlled trials of process measures for pneumonia.

\section{CONCLUSIONS}

In our study of 2076 patients hospitalized with pneumonia, we found no associations between processes of care and mortality or hospital readmission within 30 days. Although we observed small, albeit independent associations between rapid initiation of antibiotic therapy and performance of all recommended processes of care and decreased length of hospital stay, the lack of consistent evidence associating performance of pneumonia processes of care with improved patient outcomes, coupled with current high national levels of performance for these measures, casts doubts on their continued utility as quality proxies for pneumonia. In the future, researchers should conduct studies in larger populations to more definitively assess these process-outcome associations, and clinicians and policymakers need to carefully consider the next generation of quality measures for pneumonia.

\section{Acknowledgments}

Funding: Grant R01-HS10049 from the Agency for Healthcare Research and Quality. Dr Fine was supported in part from a career development award from the National Institute of Allergy and Infectious Diseases (Grant Number K24-AI001769). Dr Primack was supported in part by a Physician Faculty Scholar Award from the Robert Wood Johnson Foundation and a career development award from the National Cancer Institute (K07-CA114315).

\section{References}

1. Centers for Medicare \& Medicaid Services. [Accessed August 23, 2011] Hospital Quality Initiatives: Process of Care Measures. Available at: https://www.cms.gov/HospitalQualityInits/ 18_HospitalProcessOfCareMeasures.asp\#TopOfPage.

2. Joint Commission on Accreditation of Healthcare Organizations. [Accessed August 23, 2011] Pneumonia. Available at: http://www.jointcommission.org/pneumonia/.

3. Mandell LA, Wunderink RG, Anzueto A, et al. Infectious Diseases Society of America/American Thoracic Society consensus guidelines on the management of community-acquired pneumonia in adults. Clin Infect Dis. 2007; 44(Suppl 2):S27-S72. [PubMed: 17278083]

4. Mandell LA, Bartlett JG, Dowell SF, et al. Update of practice guidelines for the management of community-acquired pneumonia in immunocompetent adults. Clin Infect Dis. 2003; 37:1405-1433. [PubMed: 14614663]

5. Bartlett JG, Dowell SF, Mandell LA, et al. Practice guidelines for the management of communityacquired pneumonia in adults. Infectious Diseases Society of America. Clin Infect Dis. 2000; 31:347-382. [PubMed: 10987697]

6. Bernstein JM. Treatment of community-acquired pneumonia-IDSA guidelines. Infectious Diseases Society of America. Chest. 1999; 115(3 Suppl):9S-13S. [PubMed: 10084453]

7. United States Department of Health and Human Services. [Accessed August 23, 2011] Hospital Compare. Available at: http://www.hospitalcompare.hhs.gov/.

8. Joint Commission on Accreditation of Healthcare Organizations. Core Measure Sets. Available at: http://www.jointcommission.org/core_measure_set/.

9. Chassin MR, Loeb JM, Schmaltz SP, Wachter RM. Accountability measures-using measurement to promote quality improvement. N Engl J Med. 2010; 363:683-688. [PubMed: 20573915] 
10. QualityNet. [Accessed August 23, 2011] Hospital Inpatient Quality Reporting Program Overview. Available at: https://www.qualitynet.org/dcs/ContentServer?c=Page\&pagename=QnetPublic \%2FPage\%2FQnetTier2\&cid=1138115987129.

11. Bratzler DW, Nsa W, Houck PM. Performance measures for pneumonia: are they valuable, and are process measures adequate? Curr Opin Infect Dis. 2007; 20:182-189. [PubMed: 17496578]

12. Jencks SF, Huff ED, Cuerdon T. Change in the quality of care delivered to Medicare beneficiaries, 1998-1999 to 2000-2001. JAMA. 2003; 289:305-312. [PubMed: 12525231]

13. Williams SC, Schmaltz SP, Morton DJ, et al. Quality of care in U.S. hospitals as reflected by standardized measures, 2002-2004. N Engl J Med. 2005; 353:255-264. [PubMed: 16034011]

14. McGarvey RN, Harper JJ. Pneumonia mortality reduction and quality improvement in a community hospital. QRB Qual Rev Bull. 1993; 19:124-130. [PubMed: 8493027]

15. Meehan TP, Fine MJ, Krumholz HM, et al. Quality of care, process, and outcomes in elderly patients with pneumonia. JAMA. 1997; 278:2080-2084. [PubMed: 9403422]

16. Houck PM, Bratzler DW, Nsa W, et al. Timing of antibiotic administration and outcomes for Medicare patients hospitalized with community-acquired pneumonia. Arch Intern Med. 2004; 164:637-644. [PubMed: 15037492]

17. Houck PM, MacLehose RF, Niederman MS, Lowery JK. Empiric antibiotic therapy and mortality among Medicare pneumonia inpatients in 10 western states: 1993, 1995, and 1997. Chest. 2001; 119:1420-1426. [PubMed: 11348948]

18. Gleason PP, Meehan TP, Fine JM, et al. Associations between initial antimicrobial therapy and medical outcomes for hospitalized elderly patients with pneumonia. Arch Intern Med. 1999; 159:2562-2572. [PubMed: 10573046]

19. Battleman DS, Callahan M, Thaler HT. Rapid antibiotic delivery and appropriate antibiotic selection reduce length of hospital stay of patients with community-acquired pneumonia: link between quality of care and resource utilization. Arch Intern Med. 2002; 162:682-688. [PubMed: 11911722]

20. Dedier J, Singer DE, Chang Y, et al. Processes of care, illness severity, and outcomes in the management of community-acquired pneumonia at academic hospitals. Arch Intern Med. 2001; 161:2099-2104. [PubMed: 11570938]

21. Silber SH, Garrett C, Singh R, et al. Early administration of antibiotics does not shorten time to clinical stability in patients with moderate-to-severe community-acquired pneumonia. Chest. 2003; 124:1798-1804. [PubMed: 14605051]

22. Marrie TJ, Wu L. Factors influencing in-hospital mortality in community-acquired pneumonia: a prospective study of patients not initially admitted to the ICU. Chest. 2005; 127:1260-1270. [PubMed: 15821203]

23. Yealy DM, Auble TE, Stone RA, et al. The Emergency Department Community-Acquired Pneumonia Trial: methodology of a quality improvement intervention. Ann Emerg Med. 2004; 43:770-782. [PubMed: 15159711]

24. Fine MJ, Auble TE, Yealy DM, et al. A prediction rule to identify low-risk patients with community-acquired pneumonia. N Engl J Med. 1997; 336:243-250. [PubMed: 8995086]

25. Yealy DM, Auble TE, Stone RA, et al. Effect of increasing the intensity of implementing pneumonia guidelines: a randomized, controlled trial. Ann Intern Med. 2005; 143:881-894. [PubMed: 16365469]

26. Rao JNK, Scott AJ. The analysis of categorical data from complex sample surveys: chi-squared tests for goodness of fit and independence in two-way tables. J Am Stat Assoc. 1981; 76:221-230.

27. Rao JNK, Scott AJ. On chi-squared tests for multiway contingency tables with cell proportions estimated from survey data. Ann Stat. 1984; 12:46-60.

28. QualityNet. [Accessed August 23, 2011] Readmission Measures Overview. Available at: https:// www.qualitynet.org/dcs/ContentServer?c=Page\&pagename=QnetPublic\%2FPage \%2FQnetTier3\&cid=1219069855273.

29. Centers for Medicare \& Medicaid Services. Change in Composition of the National Inpatient Hospital Quality Pneumonia Measure Set. Washington, DC: Centers for Medicare \& Medicaid Services; 2008. p. 1-3. 
30. Joint Commission on Accreditation of Healthcare Organizations. [Accessed August 23, 2011] Pneumonia Care Measure Results. Available at: http://www.healthcarequalitydata.org/.

31. Freemantle N, Cleland J, Young P, et al. Beta blockade after myocardial infarction: systematic review and meta regression analysis. BMJ. 1999; 318:1730-1737. [PubMed: 10381708]

32. Effect of enalapril on survival in patients with reduced left ventricular ejection fractions and congestive heart failure. The SOLVD Investigators. N Engl J Med. 1991; 325:293-302. [PubMed: 2057034]

33. Collaborative meta-analysis of randomised trials of antiplatelet therapy for prevention of death, myocardial infarction, and stroke in high risk patients. BMJ. 2002; 324:71-86. [PubMed: 11786451] 


\section{CLINICAL SIGNIFICANCE}

- Evidence-based processes of care are considered proxies of quality in national, publicly reported quality improvement programs.

- There were no independent associations between individual processes of care or the cumulative number performed and mortality or readmission for patients with pneumonia.

- Although performing such processes of care will likely continue to represent the standard of care for pneumonia, their continued use as proxies for health care quality is uncertain. 


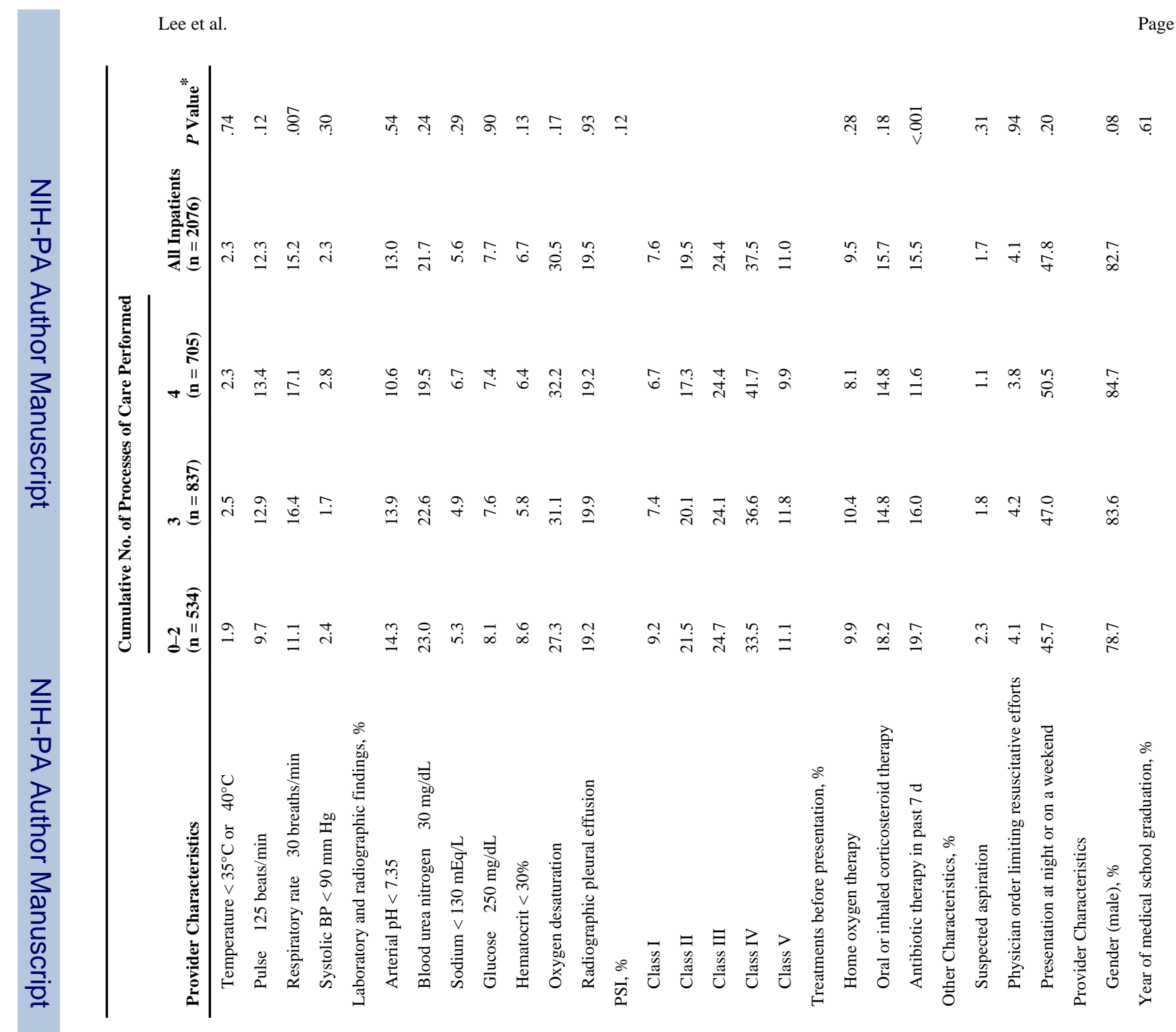

Page 12 


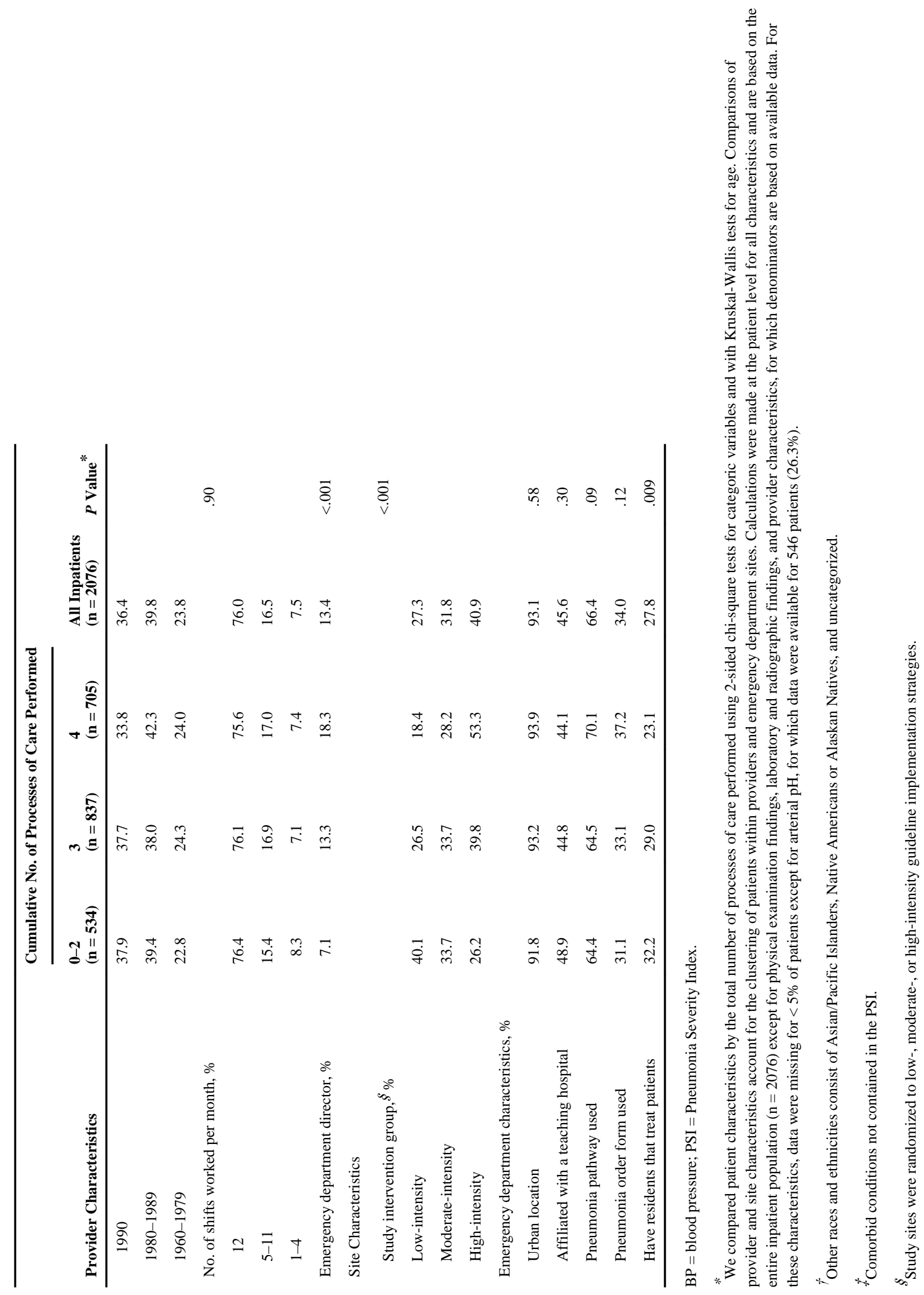


Table 2

Performance of Individual Processes of Care for Selected Patient Subgroups

\begin{tabular}{|c|c|c|c|c|}
\hline Patient Subgroups & $\begin{array}{l}\text { Oxygenation } \\
\text { Assessment (\%) }\end{array}$ & $\begin{array}{l}\text { Blood Cultures } \\
\text { Before Antibiotic } \\
\text { Administration (\%) }\end{array}$ & $\begin{array}{l}\text { Antibiotic } \\
\text { Administration } \\
\text { within } 4 \text { Hours (\%) }\end{array}$ & $\begin{array}{l}\text { Appropriate } \\
\text { Antibiotic } \\
\text { Therapy }(\%)\end{array}$ \\
\hline All inpatients $(\mathrm{N}=2076)$ & 97.6 & 63.3 & 78.6 & 63.0 \\
\hline \multicolumn{5}{|l|}{ Processes of care performed (No.) ${ }^{*}$} \\
\hline $0-2(\mathrm{~N}=534)$ & 93.4 & 25.5 & 43.8 & 15.2 \\
\hline $3(\mathrm{~N}=837)$ & 98.3 & 56.5 & 82.8 & 62.4 \\
\hline $4(\mathrm{~N}=705)^{*}$ & $100.0^{*}$ & $100.0^{*}$ & $100.0^{*}$ & $100.0^{*}$ \\
\hline \multicolumn{5}{|l|}{ Age (y) } \\
\hline$<65(\mathrm{~N}=600)$ & 97.8 & 62.8 & 79.0 & 62.3 \\
\hline 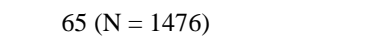 & 97.6 & 63.5 & 78.5 & 63.3 \\
\hline \multicolumn{5}{|l|}{ ICU or CCU admission } \\
\hline $\operatorname{Never}(\mathrm{N}=1792)$ & 97.7 & $62.1^{\dagger}$ & $78.3^{\dagger}$ & $64.8^{*}$ \\
\hline Day of admission $(\mathrm{N}=141)$ & 98.6 & $70.2^{\dagger}$ & $85.8^{\dagger}$ & $40.4^{t}$ \\
\hline$\geq 1 \mathrm{~d}$ after admission $(\mathrm{N}=133)$ & 96.2 & $71.4^{\dagger}$ & $72.9^{\dagger}$ & $60.9^{*}$ \\
\hline \multicolumn{5}{|l|}{ Study intervention group $\xi$} \\
\hline Low-intensity $(\mathrm{N}=566)$ & 96.3 & $53.5^{*}$ & 77.0 & $50.0 \%$ \\
\hline Moderate-intensity $(\mathrm{N}=661)$ & 99.1 & $57.6^{* t}$ & 79.7 & $59.6^{t}$ \\
\hline High-intensity $(\mathrm{N}=849)$ & 97.4 & $74.2^{f}$ & 78.8 & $74.3^{*}$ \\
\hline
\end{tabular}

$\mathrm{ICU}=$ intensive care unit; $\mathrm{CCU}=$ coronary care unit.

Patients who had all 4 processes of care performed by definition had $100 \%$ of all processes of care performed. We did not perform statistical comparisons for these patient subgroups because the individual processes of care and the cumulative number performed are interdependent.

${ }^{\dagger} P<.05$ based on a 2-sided chi-square test.

${ }^{*} P<.001$ based on a 2-sided chi-square test.

$\xi$

parisons of study intervention group use the Rao and Scott second-order corrected Pearson chi-square statistic to account for the clustering of patients within providers and sites and test for an ordinal association between study intervention group and the performance of each process of care. 


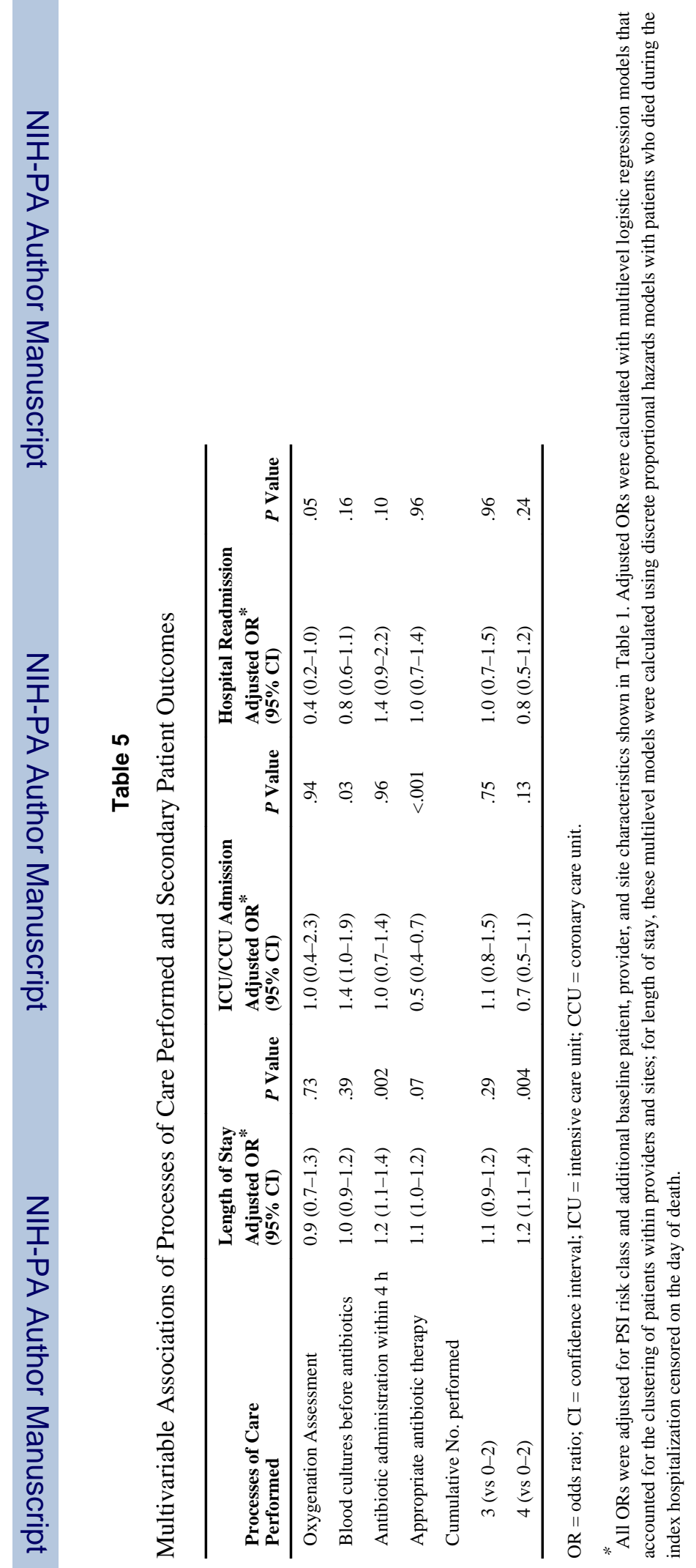

Am J Med. Author manuscript; available in PMC 2013 February 21. 